\title{
Comparision of hemodynamic changes, movement, duration of surgery and pain between lateral and supine posision after spinal anesthesia in transurethral lithotrips
}

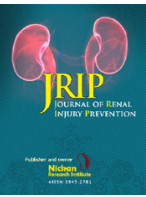

\author{
Faramarz Alibeigi ${ }^{1}{ }^{\circledR}$, Mona Hosseini $^{2}$, Mohammadreza Shabanian $^{3}$, Alireza Shabanian ${ }^{3}$, Gholamreza \\ Shabanian $^{4^{*} \mathbb{D}}$
}

${ }^{1}$ Department of Urology, Shahrekord University of Medical Sciences, Shahrekord, Iran

${ }^{2}$ Shahrekord University of Medical Sciences, Isfahan, Iran

${ }^{3}$ Isfahan University of Medical Sciences, Isfahan, Iran

${ }^{4}$ Department of Anesthesia, Shahrekord University of Medical Sciences, Shahrekord, Iran

\section{A R T I C L E I N F O}

Article Type:

Original

\section{Article History:}

Received: 10 January 2018

Accepted: 9 May 2018

Published online: 5 June 2018

Keywords:

Ureteral stone

Pain

Transurethral lithotripsy

Ureteral calculi

Urinary tract stones

Percutaneous nephrolithotomy

Staghorn calculi

\begin{abstract}
A B S T R A C T
Introduction: Patient position during surgery is an important issue that can affect vital indices. Objectives: This study was conducted to compare hemodynamic changes, movements, surgery length, and pain during transurethral lithotripsy (TUL) in supine and lateral positions.

Patients and Methods: This double-blind clinical trial was conducted on 76 patients who were candidate for TUL, randomized to two groups of 38 for each. In the lateral group, after inducing spinal anesthesia, the patients were positioned lateral and in supine group, the patients were positioned supine immediately after injecting anesthetic. Vital signs were recorded at six intervals; 5 minutes before anesthesia and 1, 5, 10, 15, and 20 minutes after anesthesia. In addition, movements and pain during surgery were assessed. Data analysis was conducted by descriptive statistics, independent $t$ test, and repeated measures ANOVA in SPSS 22.

Results: There were significant differences in changes in systolic blood pressure, diastolic blood pressure, and mean arterial pressure at different intervals between the two groups in such a way that hemodynamic stability was greater in the lateral group $(P<0.05)$. There were no significant differences in changes in heart rate at all studied intervals between the two groups $(P>0.05)$. Surgery length, movement and pain during surgery were not significantly different between the two groups $(P<0.05)$.

Conclusion: Hemodynamic changes especially blood pressure were less remarkable in patients undergoing spinal anesthesia in TUL at lateral position than those in supine position. Additionally, no significant differences in patients' movement and surgery, and surgery length between the two positions was detected.
\end{abstract}

Implication for health policy/practice/research/medical education:

Hemodynamic changes in TUL in patients undergoing spinal anesthesia is less remarkable and hemodynamic stability in the patients' blood pressure was greater at lateral position compared to supine position. But the patients' pain and movements were not different between the two positions. It is recommended to use lateral position in TUL. In addition, further studies should be conducted to investigate other factors such as the duration of recovery and respiratory rate in such patients.

Please cite this paper as: Alibeigi F, Hosseini M, Shabanian M, Shabanian A, Shabanian G. Comparision of hemodynamic changes, movement, duration of surgery and pain between lateral and supine posision after spinal anesthesia in transurethral lithotrips. J Renal Inj Prev. 2019;8(1):11-16. doi: 10.15171/jrip.2019.03.

\section{Introduction}

The prevalence of urinary tract stones is increasing worldwide (1). Urinary tract stones are one of the most important causes of kidney failure and urinary tract congestion (2). Ureteral calculi are important than other types of urinary tract stones in terms of the severity of symptoms and the speed at which kidney failure is developed (3). Transurethral lithotripsy (TUL) is one of the techniques to treat ureteral calculi. This technique is widely used in urology particularly to treat lower ureteral calculi $(4,5)$. In this technique, patient may undergo different methods of anesthesia and be positioned lithotomy (6,7). Different positions and types of anesthesia can have different effects on hemodynamic parameters 
and the quality of anesthesia and even lead to certain complications (7-9). Different patient positions are used in urology surgeries. For example, lateral position enables anesthetists to give greater sedation to the patients and to prevent the airway obstruction in some cases. Lateral position has many benefits including fewer hemodynamic complications, selective block for the limb of interest, prevention of unnecessary paralysis of the other side's limb, better movement at recovery, less incidence of urinary retention, and the patients' greater satisfaction (11). The other position is supine. Supine is a simple position, needs no instrument, and prevents the disconnection of the tracheal tube (7). However, these positions may cause certain complications and problems depending on the type of surgery or the patient's physiological conditions, and therefore affect adversely his/her vital indices $(6,7,11)$. Unilateral spinal anesthesia is used in TUL. Depending on the type of position, this technique can lead to several complications such as bradycardia, hypotension, and decreased level and rate of nerve block $(12,13)$. Besides that, a suitable position is effective on the patients' quality of vital indices during and after the surgery.

\section{Objectives}

Spinal anesthesia at supine position is conventionally used to conduct TUL, but regarding the benefits of spinal anesthesia at lateral position, we conducted the current study to comparatively investigate the possibility of this technique, the quality of anesthesia, and hemodynamic changes in supine and lateral positions in patients undergoing TUL.

\section{Patients and Methods Study population}

In this double-blind clinical trial, 76 patients from those candidates for TUL referring to the urology ward of Kashani hospital, Shahrekord (Iran) in 2014 were selected by convenience sampling. The inclusion criteria were being candidate for TUL, fulfilling the criteria of American Society of Anesthesiology (ASA) class I and II, being 20-65 years old, and volunteering to participate in the study.

The exclusion criteria are suffering from underlying disease, being at high risk, taking anti-coagulant drugs, infection at injection site, deformation, history of spine surgery, history of sensitivity to local anesthetics, and incidence any complications during surgery.

All patients were administered with $2 \mathrm{mg}$ midazolam prior to being anesthetized with hyperbaric bupivacaine $0.5 \%$. To conduct hydration, 500 cc of Ringer's lactate solution was infused to the patients on an operating table before conducting anesthetic nerve block. The anesthetic was injected by an anesthetist using a spinal needle (Quincke 25G) through L3-L4 or L4-L5 spinal segment. The patients were randomized to two groups of 38 each; supine spinal anesthesia and lateral spinal anesthesia.

Group 1; in the lateral group, the patients were spinally anesthetized as they were positioned lateral (the side that the stone had been detected in the uterus). Ten minutes later, the patients were positioned supine and TUL was performed.

Group 2; immediately after injecting the drug, the patients were spinally anesthetized in sitting position and then immediately positioned supine. In all patients, the level of selective anesthesia was considered T8-T10 that is necessary to conduct TUL (14). Information such as age, gender, substance abuse and comorbidity, surgery length, the location and the position of the stone in the ureter, American society anesthesia class, the length of nerve block, and the percentage of changes in blood pressure and heart rate was recorded in a checklist by an anesthesiology technician who was blind to the patients' grouping. In both groups, the severity of pain was measured by visual analog scale (VAS), and five minutes before inducing spinal anesthesia, the patients' blood pressure and heart rate were measured and considered to be baseline blood pressure and heart rate. In addition, 1, 5, 10, 15, and 20 minutes after bupivacaine administration, systolic and diastolic blood pressure (SBP and DBP, respectively), mean arterial blood pressure, and heart rate were recorded using the operating room monitoring system.

When SBP decreased to less than $25 \%$ of the corresponding value before anesthesia, the patient was intravenously administered with $5 \mathrm{mg}$ ephedrine, and when pain was developed during surgery, they were administered with 3 $\mu \mathrm{g} / \mathrm{kg}$ fentanyl.

\section{Ethical issues}

1) The research followed the tenets of the Declaration of Helsinki; 2) informed consent was obtained and 3) This study was approved by the Ethics Committee of Shahrekord University of Medical Sciences, Shahrekord, Iran (91-4-16 and proposal \#1151).

\section{Statistical analysis}

Data analysis was conducted by SPSS 22 using mean (standard deviation), relative frequency distribution, independent $t$ test, Mann-Whitney U test, Spearman's correlation coefficient, Chi-square test, Kruskal-Wallis test and repeated measures ANOVA and $P$ value below 0.05 was considered significant.

\section{Results}

The age range of the patients in the lateral and supine groups was 20-50 (mean: 39.6 \pm 8.9) and 20-62 (mean; $38.5 \pm 10.25$ ) years, respectively, with no significant difference according to $t$ test $(P=0.626)$. Therefore, the two groups were matched for age. Table 1 shows differences in other variables between the two groups and summarizes data on hemodynamic parameters through the study. According to Table 1, SBP was significantly different between the two groups at $5,10,15$, and 20 minutes after inducing anesthesia $(P<0.01)$ such that SBP at $5,10,15$, and 20 minutes after inducing anesthesia was 
Table 1. Comparison of the variables at baseline

\begin{tabular}{llccc}
\hline \multirow{2}{*}{ Variable } & & Group 1 & Group 2 & \multirow{2}{*}{$\boldsymbol{P}$} \\
\cline { 3 - 4 } & & No. (\%) & No. (\%) & \\
\cline { 2 - 4 } Gender & Male & $26(68.4)$ & $24(63.2)$ & \multirow{2}{*}{0.629} \\
& Female & $12(31.6)$ & $14(36.8)$ & \\
\multirow{2}{*}{ ASA Class } & $\mathrm{I}$ & $23(60.5)$ & $23(60.5)$ & \multirow{2}{*}{1} \\
& II & $15(39.5)$ & $15(39.5)$ & \\
Location of & Upper ureter & $17(44.7)$ & $13(44.7)$ & \\
the calculi & Median ureter & $10(26.3)$ & $8(21.1)$ & \multirow{2}{*}{0.64} \\
& Lower ureter & $11(28.9)$ & $8(21.1)$ & \\
Side of the & Right & $17(44.7)$ & $16(42.1)$ & \multirow{2}{*}{0.81} \\
calculi & Left & $21(55.3)$ & $22(57.9)$ & \\
\hline
\end{tabular}

significantly lower in the supine group than the lateral group. The corresponding difference was not statistically significant between the two groups five minutes before anesthesia and 1 minute after anesthesia $(P>0.05)$.

DBP was also significantly different at 10,15 , and 20 minutes after inducing anesthesia $(P<0.01)$ such that DBP at 10, 15, and 20 minutes after inducing anesthesia was significantly lower in the supine group than the lateral group. The corresponding difference was not statistically significant between the two groups five minutes before anesthesia and 1 and 5 minutes after anesthesia $(P>0.05)$ (Table 2).

Altogether, repeated measures ANOVA indicated a significant trend in SBP through the study $(P<0.05)$. Besides, this test indicated that the trend was significant in both groups $(P<0.05)$ (Figure 1$)$.

Altogether, repeated measures ANOVA indicated a significant trend in DBP through the study $(P<0.05)$. According to this test, the two groups had significantly different trends $(P<0.05)$ (Figure 2).

As Table 3 indicates, heart rates/minutes was not significantly different between the two groups at the studied intervals (5 minutes before anesthesia and 1, 5, 10, 15 , and 20 minutes after inducing anesthesia $(P>0.05)$. Besides, MAP was significantly different between the two groups at 10,15, and 20 minutes after inducing anesthesia
Table 2. Comparison of the mean values of hemodynamic parameters between the two groups

\begin{tabular}{llccc}
\hline \multirow{2}{*}{ Variable } & & Group 1 & Group 2 & \multirow{2}{*}{$\boldsymbol{P}$} \\
\cline { 3 - 4 } & & Mean \pm SD & Mean \pm SD & \\
\hline & a first & $136.71 \pm 14.79$ & $134.13 \pm 16.14$ & 0.470 \\
Systolic blood & b second & $135.47 \pm 16.58$ & $129.89 \pm 19.41$ & 0.180 \\
pressure & c third & $129.81 \pm 16$ & $117.57 \pm 16.34$ & 0.002 \\
& d fourth & $129.39 \pm 14.24$ & $115.68 \pm 13.57$ & $0.00 *^{*}$ \\
& e fifth & $131.42 \pm 13.82$ & $118.68 \pm 12.12$ & $0.000^{*}$ \\
& f sixth & $132.42 \pm 14.44$ & $119.89 \pm 11.24$ & $0.00 *^{*}$ \\
& a first & $84.15 \pm 11.52$ & $82.44 \pm 11.67$ & 0.52 \\
Diastolic blood & b second & $81.55 \pm 12.25$ & $78.76 \pm 11.78$ & 0.31 \\
pressure & c third & $78.10 \pm 11.68$ & $73.18 \pm 11.41$ & 0.067 \\
& d fourth & $79.31 \pm 9.79$ & $72.34 \pm 9.18$ & 0.002 \\
& e fifth & $80.13 \pm 9.97$ & $73.76 \pm 8.96$ & 0.005 \\
& f sixth & $81.76 \pm 9.44$ & $75.36 \pm 8.34$ & 0.003 \\
\hline
\end{tabular}

$(P<0.01)$ such that mean arterial pressure $(\mathrm{MAP})$ at 10,15 , and 20 minutes after inducing anesthesia was significantly higher in the lateral group than in the supine group. The corresponding difference was not statistically significant between the two groups 5 minutes before anesthesia and 1 and 5 minutes after anesthesia $(P>0.05)$ (Table 3$)$. Group 1; lateral, group 2; supine, a first; 5 minutes after inducing anesthesia, b second; 1 minute after inducing anesthesia, c third; 5 minutes after inducing anesthesia, $d$ fourth; 10 minutes after inducing anesthesia, e fifth; 15 minutes after inducing anesthesia, $\mathrm{f}$ sixth; 20 minutes after inducing anesthesia.

However, repeated measures ANOVA indicated no significant difference between the heartbeat trend of the two groups ( $P>0.05$; Figure 3$)$.

According to repeated measures ANOVA, the trend of MAP was significantly different between the two groups $(P<0.05$; Figure 4).

The range of surgery length in the lateral and supine

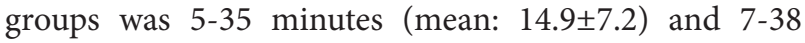
(mean: 15.6 \pm 6.6 ) minutes, respectively, with no significant difference between the two groups according to t-test $(P=0.64)$.

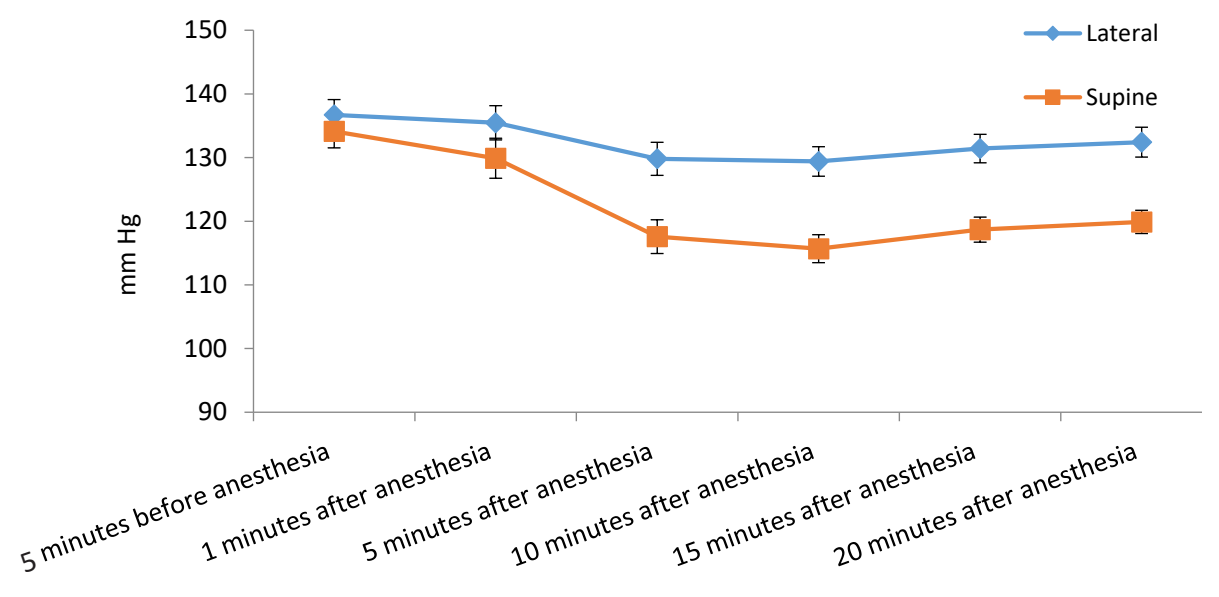

Figure 1. Patients' systolic blood pressure in tow groups during the study. 


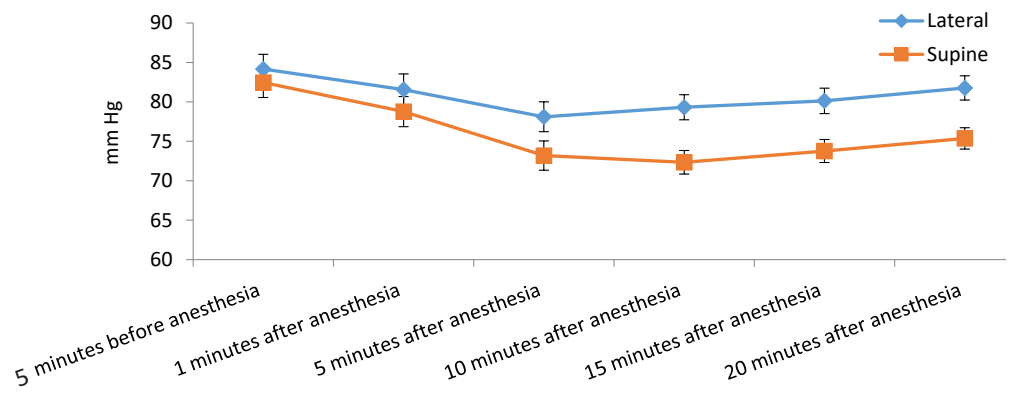

Figure 2. Patients' diastolic blood pressure in tow groups during the study.

In the lateral group, 28 (73.7\%) people had no movements during the surgery and 10 had movement during surgery. In the supine group, 27 (71.1\%) people had no movements during the surgery and 11 had movement during surgery. According to chi-square test, there was no significant difference in movement during surgery between the two groups $(P=0.79)$.

In the lateral and supine groups, according to the VAS, the range of pain was $0-3$ (mean: $0.66 \pm 0.99$ ) and $0-4$ $(0.92 \pm 1.19)$, respectively, with no significant difference between the two groups according to Mann-Whitney $\mathrm{U}$ test $(P=0.37)$. In addition, there was no significant

Table 3. Comparison of the mean values of hemodynamic parameters between the two groups

\begin{tabular}{llccc}
\hline \multirow{2}{*}{ Variable } & & Group 1 & Group 2 & \multirow{2}{*}{$\boldsymbol{P}$} \\
\cline { 3 - 4 } & & Mean \pm SD & Mean \pm SD & \\
\hline \multirow{3}{*}{ Heart rate/min } & a first & $84.13 \pm 12.53$ & $81.15 \pm 10.62$ & 0.26 \\
& b second & $85.23 \pm 13.57$ & $84.31 \pm 11.27$ & 0.74 \\
& c third & $84.86 \pm 12.59$ & $84.84 \pm 10.56$ & 0.99 \\
& d fourth & $84.10 \pm 11.50$ & $82.89 \pm 10.78$ & 0.63 \\
& e fifth & $81.63 \pm 10.05$ & $81.81 \pm 9.9$ & 0.93 \\
& f sixth & $81.68 \pm 10.40$ & $80.94 \pm 10.26$ & 0.75 \\
Mean arterial & a first & $101.64 \pm 12.17$ & $98.98 \pm 13.26$ & 0.36 \\
pressure & b second & $99.49 \pm 13.12$ & $95.72 \pm 13.49$ & 0.22 \\
& c third & $95.26 \pm 12.36$ & $87.94 \pm 12.27$ & 0.012 \\
& d fourth & $95.97 \pm 10.61$ & $86.83 \pm 9.99$ & $0.000^{*}$ \\
& e fifth & $97.10 \pm 10.6$ & $88.69 \pm 9.53$ & $0.000^{*}$ \\
& f sixth & $98.79 \pm 10.26$ & $89.93 \pm 8.61$ & $0.000^{*}$ \\
\hline
\end{tabular}

association between Spearman's correlation coefficient and surgery length $(\mathrm{r}=0.044, P=0.707)$. According to Kruskal-Wallis test, pain severity and the location of stone in the ureter were significantly associated $(P=0.006)$ such that in patients with upper ureteral calculi, the mean severity of pain was $1.2 \pm 1.2$, In those with median ureteral calculi, the mean severity of pain was $0.60 \pm 0.94$, and in those with lower ureteral calculi, the mean severity of pain was $0.26 \pm 0.65$.

\section{Discussion}

The present study was conducted to compare hemodynamic changes, movements, and pain in TUL between lateral and supine positions. A few number of studies have yet been conducted on the types of positions and hemodynamic parameters in patients with kidney stones. The present study demonstrated that in lateral position, the patients blood pressure decreased less frequently and had a relatively stable trend. However, there was no significant difference in the patients' heart rate between the two positions. A study to investigate prone and supine positions in percutaneous nephrolithotomy demonstrated that in supine position, hemodynamic changes were less remarkable and therefore supine position was better for this therapeutic procedure (15). In cesarean section, changes in blood pressure were investigated in women undergoing spinal anesthesia at different intervals. In these women, bupivacaine was

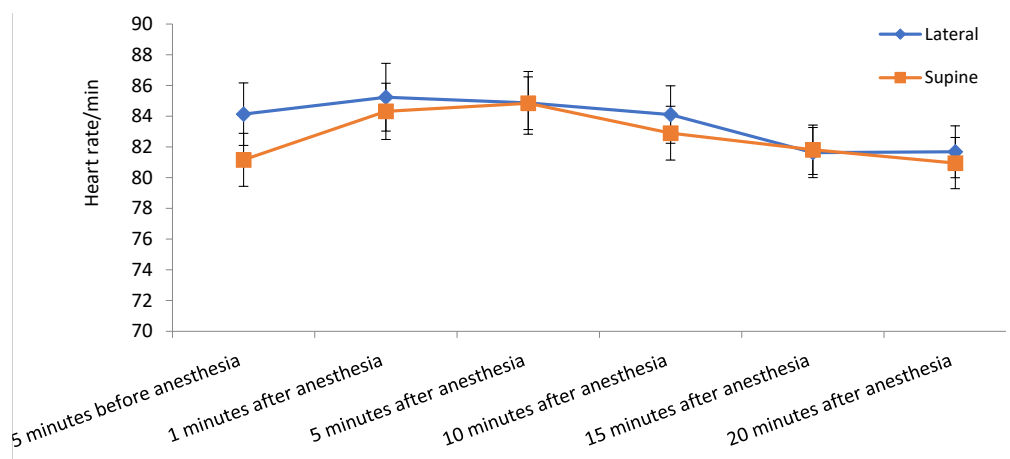

Figure 3. Patients' heart rate in tow groups during the study. 


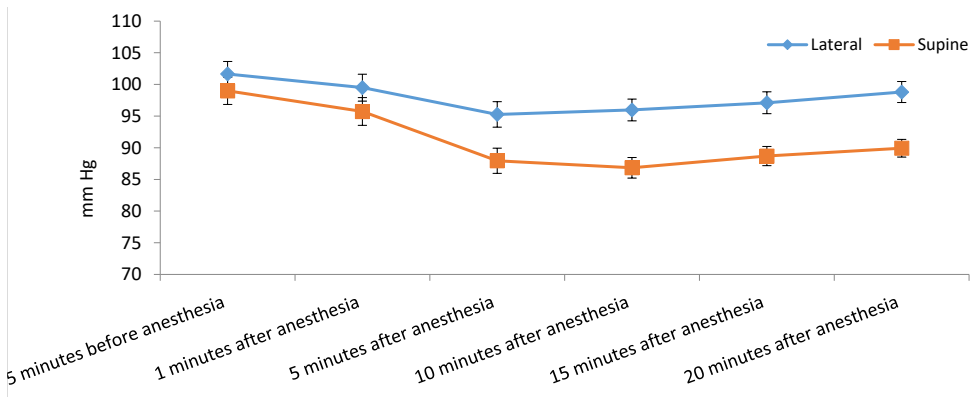

Figure 4. Patients' mean arterial pressure in tow groups during the study.

used as anesthetic, and decline in blood pressure and its changes in lateral position were less remarkable than those in the sitting position, and patients in this position had more stable hemodynamic parameters (16). The findings of the current study demonstrated that there were no significant difference in movement, surgery length, and pain between the two positions. Gokce et al compared staghorn stones between supine and prone positions and found no difference between the developed complications (17). Zhan et al also reported that the complications were not significantly different in prone and supine positions, but nephrolithotomy was conducted within a shorter time in supine position (18). A study demonstrated that the duration of percutaneous nephrolithotomy at supine position was significantly shorter than that at prone position. In the current study, supine position was concentrated on because of better urethral accessibility (19). Karimi et al compared lateral, supine, and prone positions in percutaneous nephrolithotomy. They found that supine and lateral positions were two effective methods in this type of surgery with both shorter length of surgery and fewer complications for the patients (20). However, another study demonstrated that, out of the studied variables, only surgery length was shorter at supine position, and the two positions were somehow similar for kidney stone surgeries (21). This inconsistency in the findings on surgery length can be attributed to different factors such as the type of procedure and patient characteristics, health status, and pain.

Lateral and supine positions are considered safe positions in kidney surgeries and therefore recommended for patients at high risk $(15,22)$. The patients feel more comfortable in these positions that are generally convenient and low-risk positions (23). However, the findings of a review of supine and prone positions in percutaneous nephrolithotomy showed that controversies over the type of position remain with no difference between these two positions (24). The type of surgery and patient physical conditions are determinants to select the type of position. For example, patients with staghorn calculi and obese patients develop fewer complications at supine position (25). In addition, other studies indicated that urologists should select the type of position according to the patient's conditions $(20,23)$. In this study, in TUL the patients' blood pressure was more stable at lateral position and therefore this position can be considered a suitable position for patients undergoing spinal anesthesia.

\section{Conclusion}

Hemodynamic changes in TUL in patients undergoing spinal anesthesia is less remarkable and hemodynamic stability in the patients' blood pressure was greater at lateral position compared to supine position, but the patients' pain and movements were not different between the two positions. It is recommended to use lateral position in TUL. In addition, further studies should be conducted to investigate other factors such as the duration of recovery and respiratory rate in such patients.

Limitations of the study

This is a preliminary study conducted on a limited proportion of patients. This study requires further investigation on larger samples.

\section{Acknowledgments}

This article was obtained from a research project approved by the Research and Technology Deputy of the Shahrekord University of Medical Sciences (\# 1151). Hereby, the researchers gratefully thank the women who participated in this study.

\section{Authors' contribution}

FA, GS, MH, MS and AS conceived the study and performed the experiments. FA and GS contributed to methodology and analyzed the data. All authors read, revised, and approved the final manuscript.

\section{Conflicts of interest}

There were no points of conflicts.

Ethical considerations

Ethical issues (including plagiarism, misconduct, data fabrication, falsification, double publication or submission, redundancy) have been completely observed by the authors.

Funding/Support

This project supervised by research deputy Shahrekord 
University of Medical Sciences without any Grant (\# 1151).

\section{References}

1. Romero V, Akpinar H, Assimos DG. Kidney Stones: A Global Picture of Prevalence, Incidence, and Associated Risk Factors. Rev Urol. 2010;12:e86-e96.

2. Mao S, Jiang H, Wu Z, Fang Z, Xia G, Ding Q. Urolithiasis: the most risk for nephrectomy in nonrenal tumor patients. J Endourol. 2012;26:1356-60. doi: 10.1089/end.2012.0080.

3. Kovacevic L, Lu H, Caruso JA, Lakshmanan Y. Renal Tubular Dysfunction in Pediatric Urolithiasis: Proteomic Evidence. Urology. 2016;92:100-5. doi: 10.1016/j.urology.2016.02.003.

4. Shigemura K, Yasufuku T, Yamashita M, Arakawa S, Fujisawa M. Efficacy of combining flexible and rigid ureteroscopy for transurethral lithotripsy. Kobe J Med Sci. 2010;56:E24-8.

5. Nishimura Y, Moriya K, Mitsui T, Kitta T, Kanno Y, Kon M, et al. [Transurethral Ureterolithotripsy for Upper Urinary Tract Stone in Small Children Weighning Around 10kg]. Nihon Hinyokika Gakkai Zasshi. 2015;106:285-8.

6. Kim HY, Lee MJ, Kim M-N, Kim J-S, Lee WS, Lee KC. Effect of position changes after spinal anesthesia with low-dose bupivacaine in elderly patients: sensory block characteristics and hemodynamic changes. Korean J Anesthesiol. 2013;64:234-9. doi: 10.4097/kjae.2013.64.3.234.

7. Rozet I, Vavilala MS. Risks and benefits of patient positioning during neurosurgical care. Anesthesiol Clin. 2007;25:631-x. doi: 10.1016/j.anclin.2007.05.009.

8. Cormio L, Annese P, Corvasce T, De Siati M, Turri FP, Lorusso F, et al. Percutaneous nephrostomy in supine position. Urology. 2007;69:377-80. doi: 10.1016/j.urology.2007.01.020.

9. Schonauer C, Bocchetti A, Barbagallo G, Albanese V, Moraci A. Positioning on surgical table. Eur Spine J. 2004;13:S50-S5. doi:10.1007/s00586-004-0728-y.

10. Coda B, Barash P, Cullen B, Stoelting R, Cahalan M, Stock M. Clinical anesthesia. Philadelphia: Lippicott Williams \& Wilkins; 2006. p. 691-717.

11. Kwee MM, Ho Y-H, Rozen WM. The Prone Position During Surgery and its Complications: A Systematic Review and Evidence-Based Guidelines. Int Surg. 2015;100:292-303. doi:10.9738/INTSURG-D-13-00256.1.

12. Imbelloni LE. Spinal hemianesthesia: Unilateral and posterior. Anesth Essays Res. 2014;8:270-6. doi:10.4103/02591162.143108

13. Kilinc LT, Sivrikaya GU, Eksioglu B, Hanci A, Dobrucali H. Comparison of unilateral spinal and continous spinal anesthesia for hip surgery in elderly patients. Saudi J Anaesth. 2013;7:404-9. doi:10.4103/1658-354X.121054.

14. Barash PG. Clinical anesthesia. Philadelphia: Lippincott Williams \& Wilkins; 2009.
15. Khoshrang H, Falahatkar S, Ilat S, Akbar MH, Shakiba M, Farzan A, et al. Comparative Study of Hemodynamics electrolyte and metabolic changes during prone and complete supine percutaneous nephrolithotomy. Nephrourol Mon. 2012;4:622-8. doi: 10.5812/numonthly.4099.

16. Obasuyi BI, Fyneface-Ogan S, Mato CN. A comparison of the haemodynamic effects of lateral and sitting positions during induction of spinal anaesthesia for caesarean section. Int J Obstet Anesth. 2013;22:124-8. doi: 10.1016/j. ijoa.2012.12.005.

17. Gokce MI, Ibis A, Sanci A, Akinci A, Bagci U, Agaoglu EA, et al. Comparison of supine and prone positions for percutaneous nephrolithotomy in treatment of staghorn stones. Urolithiasis. 2017;45:603-608. doi: 10.1007/s00240017-0977-y.

18. Zhan HL, Li ZC, Zhou XF, Yang F, Huang JF, Lu MH. Supine lithotomy versus prone position in minimally invasive percutaneous nephrolithotomy for upper urinary tract calculi. Urol Int. 2013;91:320-5. doi:10.1159/000351337.

19. Falahatkar S, Moghaddam AA, Salehi M, Nikpour S, Esmaili F, Khaki N. Complete supine percutaneous nephrolithotripsy comparison with the prone standard technique. J Endourol. 2008;22:2513-7. doi: 10.1089/end.2008.0463.

20. Karami H, Mohammadi R, Lotfi B. A study on comparative outcomes of percutaneous nephrolithotomy in prone, supine, and flank positions. World J Urol. 2013;31:1225-30. doi: 10.1007/s00345-012-0889-y.

21. De Sio M, Autorino R, Quarto G, Calabro F, Damiano R, Giugliano F, et al. Modified supine versus prone position in percutaneous nephrolithotomy for renal stones treatable with a single percutaneous access: a prospective randomized trial. Eur Urol. 2008;54:196-202. doi: 10.1016/j. eururo.2008.01.067.

22. Vicentini FC, Gomes CM, Danilovic A, Neto EAC, Mazzucchi E, Srougi M. Percutaneous nephrolithotomy: Current concepts. Indian J Urol. 2009;25:4-10. doi: 10.4103/09701591.44281.

23. Karaolides T, Moraitis K, Bach C, Masood J, Buchholz N. Positions for percutaneous nephrolithotomy: Thirtyfive years of evolution. Arab J Urol. 2012;10:307-16. doi: 10.1016/j.aju.2012.06.005.

24. Mak DKC, Smith Y, Buchholz N, El-Husseiny T. What is better in percutaneous nephrolithotomy - Prone or supine? A systematic review. Arab J Urol. 2016;14:101-7. doi:10.1016/j. aju.2016.01.005.

25. de la Rosette JJ, Tsakiris P, Ferrandino MN, Elsakka AM, Rioja J, Preminger GM. Beyond prone position in percutaneous nephrolithotomy: a comprehensive review. Eur Urol. 2008;54:1262-9. doi:10.1016/j.eururo.2008.08.012.

Copyright $\odot 2019$ The Author(s); Published by Nickan Research Institute. This is an open-access article distributed under the terms of the Creative Commons Attribution License (http://creativecommons.org/licenses/by/4.0), which permits unrestricted use, distribution, and reproduction in any medium, provided the original work is properly cited. 